\title{
Usuarios de teleasistencia domiciliaria: enfermedades y medicación
}

\author{
A. Maseda ${ }^{\mathrm{a}}$, S.Martínez-Isasi ${ }^{\mathrm{b}}$, L. Lorenzo-López ${ }^{\mathrm{a}}$, A. Buján ${ }^{\mathrm{a}}$, L. Regueiro- \\ Folgueira $^{\mathrm{a}}$, J.C. Millán-Calenti ${ }^{\mathrm{a}}$
}

${ }^{a}$ Universidade da Coruña, Grupo de Investigación en Gerontología, Instituto de Investigación Biomédica de A Coruña (INIBIC), Complexo Hospitalario Universitario de A Coruña (CHUAC), SERGAS, A Coruña, España

${ }^{b}$ Universidade da Coruña, Unidad de Investigación de Salud y Podología, Departamento de Ciencias de la Salud, A Coruña, España

Objetivos: Los servicios de teleasistencia a domicilio para personas mayores han evolucionado, desde los clásicos simplemente de telealarma hasta los actuales, que permiten, además del apoyo social, el seguimiento y control de aspectos de salud. El objetivo de este trabajo ha sido conocer el perfil sociosanitario de los usuarios de un servicio de teleasistencia.

Métodos: Se realizó estudio transversal retrospectivo, a partir de la población $(\mathrm{n}=850)$ de personas mayores (65 o más años) usuarias del servicio de teleasistencia domiciliaria (telealarma telefónica) de la Cruz Roja en la ciudad de A Coruña. Un total de 742 (85,3\%) personas cumplieron los criterios de inclusión y participaron en el estudio.

Resultados: Las personas mayores, como media, llevaban casi 5 años como usuarias del servicio, con una media de edad de $83,3 \pm 6,6$ años. La mayor parte $(85,8 \%)$ eran mujeres y personas que vivían solas $(78,3 \%)$. Las personas mayores usuarias de teleasistencia respondían al criterio de polifarmacia $(59,4 \%)$ por el consumo de un número elevado de medicamentos $(5,6 \pm 3,0)$, principalmente vinculados al sistema cardiovascular (75\%) y al nervioso $(65,2 \%)$. En el caso de las enfermedades, en su mayoría tenían una sola afección, principalmente hipertensión $(51,1 \%)$ o enfermedades reumáticas (44\%). Además, el 51,3\% tenían discapacidad, principalmente física, presentando además el 17,9\% dependencia en al menos una actividad básica de la vida diaria. La presencia de hipertensión, los síntomas ansioso-depresivos y la insuficiencia cardiaca se identificaron como determinantes de la polifarmacia.

Conclusiones: de los datos obtenidos, entendemos que a la hora de implementar los servicios de teleasistencia han de tenerse en cuenta, además de la problemática social, aspectos referidos a la salud; determinados, en nuestro caso, por el importante porcentaje de sujetos afectos de polifarmacia. Solamente si tenemos en cuenta dichas características sociosanitarias, la eficacia de los recursos a domicilio será efectiva y permitirá conseguir el objetivo del mismo: mantener a la persona mayor por más tiempo en su propio domicilio.

Este trabajo ha sido financiado por la Xunta de Galicia (ED431C2017/49 y Red FrailNet IN607C 2016/08). 